\title{
Universal attractor for nonlinear one-dimensional compressible and radiative MHD flow
}

Xin Liu*

"Correspondence:
xinliu120@suibe.edu.cn
Department of Applied
Mathematics, School of Business
Information Management,
Shanghai University of International
Business and Economics, Songjiang,
Shanghai 201620, P.R. China

\section{"Correspondence:}

xinliu120@suibe.edu.c

Information Management,

Business and Economics, Songjiang,

Shanghai 201620, P.R. China

\begin{abstract}
This paper is concerned with the existence of universal attractors in $H_{+}^{i}(i=1,2)$ for one-dimensional compressible and radiative magnetohydrodynamics equations in a bounded domain $\Omega:=(0,1)$. In this paper, the author extends the results in (Qin et al. in J. Differ. Equ. 253:1439-1488, 2012).
\end{abstract}

MSC: 35B45; 35L65; 35Q60; 76N10

Keywords: magnetohydrodynamics (MHD); thermal radiation; absorbing set; universal attractor

\section{Introduction}

In this paper, we study the existence of universal attractors to the one-dimensional compressible thermally radiative magnetohydrodynamic equations.

Magnetohydrodynamics (MHD) is concerned with the study of the interaction between magnetic fields and fluid conductors of electricity. The applications of magnetohydrodynamics cover a very wide range of physical areas from liquid metals to cosmic plasmas, for example, the intensely heated and ionized fluids in an electromagnetic field in astrophysics, geophysics, high-speed aerodynamics, and plasma physics. In addition to these situations, we also take into account the effect of the radiation field. The motion mentioned above is described by the following equations in the Lagrangian coordinate system:

$$
\begin{aligned}
& \tau_{t}-u_{x}=0, \\
& u_{t}+\left(p+\frac{1}{2}|\mathbf{b}|^{2}\right)_{x}=\left(\frac{\lambda u_{x}}{\tau}\right)_{x} \\
& \mathbf{w}_{t}-\mathbf{b}_{x}=\left(\frac{\mu \mathbf{w}_{x}}{\tau}\right)_{x} \\
& (\tau \mathbf{b})_{t}-\mathbf{w}_{x}=\left(\frac{\nu \mathbf{b}_{x}}{\tau}\right)_{x} \\
& E_{t}+\left(u\left(p+\frac{1}{2}|\mathbf{b}|^{2}\right)-\mathbf{w} \cdot \mathbf{b}\right)_{x}=\left(\frac{\lambda u u_{x}+\mu \mathbf{w} \cdot \mathbf{w}_{x}+\nu \mathbf{b} \cdot \mathbf{b}_{x}+\kappa \theta_{x}}{\tau}\right)_{x},
\end{aligned}
$$

(c) 2015 Liu. This article is distributed under the terms of the Creative Commons Attribution 4.0 International License (http://creativecommons.org/licenses/by/4.0/), which permits unrestricted use, distribution, and reproduction in any medium, provided you give appropriate credit to the original author(s) and the source, provide a link to the Creative Commons license, and indicate if changes were made. 
here $\tau=\frac{1}{\rho}$ denotes the specific volume, $u \in \mathbf{R}$ the longitudinal velocity, $\mathbf{w} \in \mathbf{R}^{2}$ the transverse velocity, $\mathbf{b} \in \mathbf{R}^{2}$ the transverse magnetic field, and $\theta$ the temperature, $p=p(\tau, \theta)$ the pressure, and $e=e(\tau, \theta)$ the internal energy; $\lambda$ and $\mu$ are the bulk and the shear viscosity coefficients, respectively, $v$ is the magnetic diffusivity acting as a magnetic diffusion coefficient of the magnetic field, $k=k(\tau, \theta)$ is the heat conductivity, $E$ is given by

$$
E=e+\frac{1}{2}\left(u^{2}+|\mathbf{w}|^{2}\right)+\frac{1}{2} \tau|\mathbf{b}|^{2} .
$$

For the constitutive relations, we consider (see, e.g., [1]) the Stefan-Boltzmann model, i.e., the pressure $p(\tau, \theta)$, internal energy $e(\tau, \theta)$, and the thermo-radiative flux $Q(\tau, \theta)$ take the following forms, respectively:

$$
p(\tau, \theta)=\frac{R \theta}{\tau}+\frac{a}{3} \theta^{4}, \quad e(\tau, \theta)=C_{\nu} \theta+a \tau \theta^{4}, \quad Q(\tau, \theta)=Q_{F}+Q_{R}=-\kappa \theta_{x},
$$

where $R>0$ is the perfect gas constant, $C_{v}>0$ is the specific heat at constant volume, $a>0$ is a constant and the heat conductivity $\kappa(\tau, \theta)>0$ is a function of $\tau$ and $\theta$. As initial and boundary conditions, we consider

$$
\begin{aligned}
& \left.(\tau, u, \mathbf{w}, \mathbf{b}, \theta)\right|_{t=0}=\left(\tau_{0}, u_{0}, \mathbf{w}_{0}, \mathbf{b}_{0}, \theta_{0}\right)(x), \quad x \in \bar{\Omega}=[0,1], \\
& \left.\left(u, \mathbf{w}, \mathbf{b}, \theta_{x}\right)\right|_{\partial \Omega}=0 .
\end{aligned}
$$

Before starting and proving our results, let us first recall the related results in the literature. For the one-dimensional ideal gas, i.e.,

$$
e=C_{\nu} \theta, \quad \sigma=-\frac{R \theta}{\tau}+\frac{\mu}{\tau} u_{x}, \quad Q=-\kappa \frac{\theta_{x}}{\tau}, \quad \mathbf{w}=\mathbf{b} \equiv \mathbf{0},
$$

with suitable positive constants $C_{v}, R$, Kazhikhov [2, 3], Kazhikhov and Shelukhin [4], Kawashima and Nishida [5] established the existence of global smooth solutions. Zheng and Qin [6] proved the existence of maximal attractors in $H^{i}(i=1,2)$. However, under very high temperatures and densities, the constitutive relations (1.9) become inadequate. Thus a more realistic model would be a linearly viscous gas (or Newtonian fluid),

$$
\sigma\left(\tau, \theta, u_{x}\right)=-p(\tau, \theta)+\frac{\mu(\tau, \theta)}{\tau} u_{x}
$$

satisfying Fourier's law of heat flux,

$$
Q\left(\tau, \theta, \theta_{x}\right)=-\frac{\kappa(\tau, \theta)}{\tau} \theta_{x}
$$

whose internal energy $e$ and pressure $p$ are coupled by the standard thermodynamical relation

$$
e_{\tau}(\tau, \theta)=-p(\tau, \theta)+\theta p_{\theta}(\tau, \theta) \text {. }
$$

In this case, Kawohl [7] and Jiang [8] obtained the existence of global solutions to $1 D$ viscous heat-conductive real gas with different growth assumptions on the pressure $p$, internal energy $e$, and heat conductivity $\kappa$ in terms of temperature. Qin [9] established the regularity and asymptotic behavior of global solutions with more general growth assumptions on $p, e, \kappa$ than those in $[7,8]$. 
For the radiative and reactive gas, Ducomet [1] established the global existence and exponential decay in $H^{1}$ of smooth solutions. Umehara and Tani [10] and Qin et al. [11] proved the global existence of smooth solutions for a self-gravitating radiative and reactive gas.

For the non-radiative MHD flows (i.e., $a \equiv 0$ ), there have been a number of studies under various conditions by several authors (see, e.g., [12-18]). The existence and uniqueness of local smooth solutions was first obtained in [17], moreover, the existence of global smooth solutions with small smooth initial data was shown in [19]. Under the technical condition that $\kappa(\rho, \theta)$ satisfies

$$
0<C^{-1}\left(1+\theta^{q}\right) \leq \kappa(\rho, \theta) \leq C\left(1+\theta^{q}\right)
$$

for $q \geq 2$, Chen and Wang [12] proved the existence and continuous dependence of global strong solutions with large initial data satisfying

$$
0<\inf \rho_{0} \leq \rho_{0}(x) \leq \sup \rho_{0}<\infty, \quad \rho_{0}, u_{0}, \mathbf{w}_{0}, \mathbf{b}_{0}, \theta_{0} \in H^{1}(\Omega), \quad \theta_{0}(x)>0 .
$$

Chen and Wang [13] also investigated a free boundary problem with general large initial data. Wang [18] established the existence of large solutions to the initial-boundary value problem for planar magnetohydrodynamics. Under the technical condition upon $\kappa(\rho)$

$$
\kappa(\rho, \theta) \equiv \kappa(\rho)>\frac{C}{\rho}
$$

Fan, Jiang and Nakamura [15] investigated the uniqueness of the weak solutions of MHD with Lebesgue initial data. Fan, Jiang and Nakamura [16] also considered a onedimensional plane MHD compressible flow, and proved that as the shear viscosity goes to zero, global weak solutions converge to a solution of the original equations with zero shear viscosity. The uniqueness and continuous dependence of weak solutions for the Cauchy problem have been proved by Hoff and Tsyganov [14].

For compressible and radiative MHD flow (i.e., $a>0$ ), the author and his colleagues [20, $21]$ established the global existence and exponential stability of solutions. For compressible and radiative MHD flow (i.e., $a>0$ ) with self-gravitation, Ducomet and Feireisl [22] proved the existence of global-in-time solutions of this problem with arbitrarily large initial data and conservative boundary conditions on a bounded spatial domain in $\mathbf{R}^{3}$. Under the technical condition that $\kappa(\rho, \theta)$ satisfies

$$
k_{1}\left(1+\theta^{q}\right) \leq \kappa(\rho, \theta), \quad\left|\kappa_{\rho}(\rho, \theta)\right| \leq k_{2}\left(1+\theta^{q}\right),
$$

for some $q>\frac{5}{2}$, Zhang and Xie [23] investigated the existence of global smooth solutions to this problem. However, the large-time behavior is still open even for the nonself-gravitative case, i.e., (1.1)-(1.8). In this paper, we obtained the existence of universal attractors in $H_{+}^{i}(i=1,2)$ (see below for their definitions) for one-dimensional compressible and radiative magnetohydrodynamics equations. Before starting the research, let us first explain some mathematical difficulties in studying this problem.

Firstly, for physical reasons, the special volume $\tau$ and the absolute temperature $\theta$ should be positive for all time. These constraints give rise to some severe mathematical difficulties. For instance, we must work on incomplete metric spaces $H_{+}^{1}$ and $H_{+}^{2}, H_{+}^{2} \subset H_{+}^{1}$, which are the usual Sobolev spaces with these constrains. 
Secondly, since the universal attractor is just the $\omega$-limit set of an absorbing set in weak topology, the requirement of completeness of the spaces is needed. To overcome this severe mathematical difficulty, we restrict ourselves to a sequence of closed subspaces $H_{+}^{1}$ and $H_{+}^{2}$. It turns out that it is very crucial to prove that the orbit starting from any bounded set of this closed subspace will re-enter this subspace and stay there after a finite time, which should be uniform with respect to all orbits starting from a bounded set, otherwise, there is no ground to talk about the existence of an absorbing set and a (maximal) universal attractor in this subspace. The proof of the above fact becomes an essential part of this paper and it will be done by use of delicate $a$ priori estimates.

Thirdly, the total mass and the total energy are conserved. These conservations indicate that there can be no absorbing set for initial data varying in the whole space. Instead, we should rather consider the dynamics in a sequence of closed subspaces defined by some parameters. In this regard, the situation is quite similar to those encountered for the single Cahn-Hilliard equation in the isothermal case (see Temam [24], Zheng and Qin [6] and Qin [9]). Therefore, one of the key issues is how to choose these closed subspaces.

Fourthly, (1.1)-(1.8) is a hyperbolic-parabolic coupled system. It turns out that in general the orbit is not compact. In order to prove the existence of a maximal attractor by the theory presented by Temam in [24], we have either to show the uniform compactness of the orbit of semigroup $S(t)$ for large time or to show that one can decompose $S(t)$ into two parts, $S_{1}(t)$ and $S_{2}(t)$, with $S_{1}(t)$ being uniformly compact for large time and $S_{2}(t)$ going to zero uniformly. Moreover, since our system is quasilinear, the usual way of decomposition of $S(t)$ into two parts for a semilinear system does not seem feasible. To overcome this difficulty, we will adopt an approach motivated by the ideas in Ghidaglia [25] (see also, Lemma 2.1).

Finally, (1.1)-(1.8) are complicated, it turns out that very delicate estimates are needed. We define two spaces as follows:

$$
\begin{aligned}
H_{+}^{1}= & \left\{(\tau, u, \mathbf{w}, \mathbf{b}, \theta) \in\left(H^{1}[0,1]\right)^{7}: \tau(x)>0, \theta(x)>0, x \in[0,1],\right. \\
& u(0)=u(1)=0, \mathbf{w}(0)=\mathbf{w}(1)=\mathbf{b}(0)=\mathbf{b}(1)=\mathbf{0}\}, \\
H_{+}^{2}= & \left\{(\tau, u, \mathbf{w}, \mathbf{b}, \theta) \in\left(H^{2}[0,1]\right)^{7}: \tau(x)>0, \theta(x)>0, x \in[0,1],\right. \\
& u(0)=u(1)=0, \mathbf{w}(0)=\mathbf{w}(1)=\mathbf{b}(0)=\mathbf{b}(1)=\mathbf{0}, \\
& \left.\theta^{\prime}(0)=\theta^{\prime}(1)=0\right\},
\end{aligned}
$$

which become two metric spaces when equipped with the metrics induced from the usual normal. In the above, $H^{1}, H^{2}$ are the usual Sobolev spaces.

Let

$$
\begin{aligned}
H_{\delta}^{i}= & \left\{(\tau, u, \mathbf{w}, \mathbf{b}, \theta) \in H_{+}^{i}: \delta_{2} \leq \int_{0}^{1} \tau(x, t) d x=\int_{0}^{1} \tau_{0}(x) d x \leq \delta_{3},\right. \\
& \delta_{6} \leq \int_{0}^{1} E(x, t) d x \leq \delta_{7}, \delta_{4} \leq \theta(x, t) \leq \delta_{5}, \delta_{2} / 2 \leq \tau(x, t) \leq 2 \delta_{3}, \\
& \left.\Phi(t)+\int_{0}^{t} V(s) d s \leq \delta_{1}\right\}, \quad i=1,2,
\end{aligned}
$$


where

$$
\begin{aligned}
& \Phi(t)=\int_{0}^{1}\left[C_{v}(\theta-\log \theta-1)+R(\tau-\log \tau-1)\right](x, t) d x, \\
& V(t)=\int_{0}^{1}\left(\frac{\kappa \theta_{x}^{2}}{\tau \theta^{2}}+\frac{\lambda u_{x}^{2}+\mu\left|\mathbf{w}_{x}\right|^{2}+v\left|\mathbf{b}_{x}\right|^{2}}{\tau \theta}\right)(x, t) d x, \\
& E(x, t)=e(\tau, \theta)+\frac{1}{2}\left(u^{2}+|\mathbf{w}|^{2}\right)+\frac{1}{2} \tau|\mathbf{b}|^{2}=C_{v} \theta+a \tau \theta^{4}+\frac{1}{2}\left(u^{2}+|\mathbf{w}|^{2}\right)+\frac{1}{2} \tau|\mathbf{b}|^{2}, \\
& \delta_{1} \in \mathbf{R}^{3}, \quad 0<\delta_{2}<\delta_{3}, \quad 0<\delta_{4}<\delta_{5}, \quad 0<\delta_{6}<\delta_{7} .
\end{aligned}
$$

The notation in this paper will be chosen as follows:

$L^{p}, 1 \leq p \leq+\infty, W^{m, p}, m \in N, H^{1}=W^{1,2}, H_{0}^{1}=W_{0}^{1,2}$ denote the usual (Sobolev) spaces on $[0,1]$. In addition, $\|\cdot\|_{B}$ denotes the norm in the space $B$, we also put $\|\cdot\|=\|\cdot\|_{L^{2}[0,1]}$. The constants $C_{i}(i=1,2)$ stand for universal positive constants depending only on the $H^{i}$ norm of the initial data, $\min _{x \in[0,1]} \theta_{0}$ and $\min _{x \in[0,1]} \tau_{0}$. $C_{\delta}$ stands for the universal positive constant, but independent of any length of time. $C_{B_{i}, \delta}$ denotes the universal positive constant depending only on $\delta_{i}(i=1, \ldots, 7)$, the $H_{+}^{i}$ norm of the initial data $\left(\tau_{0}, u_{0}, \mathbf{w}_{0}, \mathbf{b}_{0}, \theta_{0}\right)$, $\min _{x \in[0,1]} \theta_{0}$, and $\min _{x \in[0,1]} \tau_{0}$.

Now we are in a position to state our main results.

Theorem 1.1 Assume that e, $p$, and $Q$ are $C^{2}$ functions satisfying (1.6) on $0<\tau<\infty$ and $0 \leq \theta<\infty$. Then the unique generalized global solution $(\tau, u, \mathbf{w}, \mathbf{b}, \theta)$ to problem (1.1)-(1.8) defines a nonlinear $C_{0}$-semigroup $S(t)$ on $H_{+}^{1}$. Moreover, for any $\delta_{i}(i=1, \ldots, 7)$, it possesses in $H_{\delta}^{1}$ a universal (maximal) attractor $\mathcal{A}_{1, \delta}$.

Theorem 1.2 Assume that e, $p$, and $Q$ are $C^{3}$ functions satisfying (1.6) on $0<\tau<\infty$ and $0 \leq \theta<\infty$. Then the unique generalized global solution $(\tau, u, \mathbf{w}, \mathbf{b}, \theta)$ to problem (1.1)-(1.8) defines a nonlinear $C_{0}$-semigroup $S(t)$ on $H_{+}^{2}$. Moreover, for any $\delta_{i}(i=1, \ldots, 7)$, it possesses in $H_{\delta}^{2}$ a universal (maximal) attractor $\mathcal{A}_{2, \delta}$.

Remark 1.1 See Ghidaglia [25] and Qin [9] for the definition of universal (maximal) attractor.

\section{An absorbing set in $H^{1}$}

In this section we will prove the existence of an absorbing ball in $H_{\delta}^{1}$. Throughout this section we assume that the initial data belong to a bounded set of $H_{\delta}^{1}$. First, we have to prove that the orbit starting from any bounded set of $H_{\delta}^{1}$ will re-enter $H_{\delta}^{1}$ and stay there after a finite time, which should be a uniform with respect to all orbits starting from that bounded set.

Lemma 2.1 Let $H_{1}, H_{2}, H_{3}$ be three Banach spaces verifying the following conditions:

(1) the embeddings $H_{3} \rightarrow H_{2}$ and $H_{2} \rightarrow H_{1}$ are compact;

(2) there are $\mathrm{C}_{0}$-semigroup $\mathrm{S}(\mathrm{t})$ on $\mathrm{H}_{2}$ and $\mathrm{H}_{3}$ which map $\mathrm{H}_{2}, \mathrm{H}_{3}$ into $\mathrm{H}_{2}, \mathrm{H}_{3}$, respectively, and for any $t>0, S(t)$ are continuous (nonlinear) operators on $\mathrm{H}_{2}, \mathrm{H}_{3}$ respectively;

(3) the semigroup $S(t)$ on $H_{3}$ possesses a bounded absorbing set in $H_{3}$; then there is a weak universal attractor $\mathcal{A}_{3}$ in $\mathrm{H}_{3}$. 
If, further, the following conditions are valid:

(4) the semigroup $S(t)$ on $\mathrm{H}_{2}$ possesses a bounded absorbing set in $\mathrm{H}_{2}$;

(5) for any $t>0, S(t)$ is continuous on bounded sets of $\mathrm{H}_{2}$ for the topology of the norm of $H_{1}$, then there is a weak universal attractor $\mathcal{A}_{2}$ in $\mathrm{H}_{2}$.

Proof See, e.g., Ghidaglia [25].

Lemma 2.2 Assume that the initial data $\left(\tau_{0}, u_{0}, \mathbf{w}_{0}, \mathbf{b}_{0}, \theta_{0}\right) \in H_{+}^{1}$, and compatibility conditions are satisfied, and the heat conductivity $\kappa$ is a $C^{2}$ function on $0<\tau<\infty$ and $0 \leq \theta<\infty$ and satisfies the growth condition

$$
k_{1}\left(1+\theta^{q}\right) \leq \kappa(\tau, \theta) \leq k_{2}\left(1+\theta^{q}\right), \quad\left|\kappa_{\tau}\right|+\left|\kappa_{\tau \tau}\right| \leq k_{2}\left(1+\theta^{q}\right), \quad q>2,
$$

with positive constants $k_{1} \leq k_{2}$, and there exists a constant $\varepsilon_{0}>0$ such that $\bar{\tau}=\int_{0}^{1} \tau_{0} d x \leq$ $\varepsilon_{0}$. Then problem (1.1)-(1.8) admits a unique global solution $(\tau, u, \mathbf{w}, \mathbf{b}, \theta) \in H_{+}^{1}$ verifying

$$
0<C_{1}^{-1} \leq \tau(x, t) \leq C_{1}, \quad 0<C_{1}^{-1} \leq \theta(x, t) \leq C_{1}, \quad \forall(x, t) \in[0,1] \times[0, \infty)
$$

and for any $t>0$

$$
\begin{aligned}
& \|\tau(t)-\bar{\tau}\|_{H^{1}}^{2}+\|u(t)\|_{H^{1}}^{2}+\|\mathbf{w}(t)\|_{H^{1}}^{2}+\|\mathbf{b}(t)\|_{H^{1}}^{2}+\|\theta(t)-\bar{\theta}\|_{H^{1}}^{2} \\
& \quad+\int_{0}^{t}\left(\|\tau-\bar{\tau}\|_{H^{1}}^{2}+\|u\|_{H^{2}}^{2}+\|\mathbf{w}\|_{H^{2}}^{2}+\|\mathbf{b}\|_{H^{2}}^{2}+\|\theta-\bar{\theta}\|_{H^{2}}^{2}+\left\|u_{t}\right\|^{2}\right. \\
& \left.\quad+\left\|\mathbf{w}_{t}\right\|^{2}+\left\|\mathbf{b}_{t}\right\|^{2}+\left\|\theta_{t}\right\|^{2}\right)(s) d s \leq C_{1},
\end{aligned}
$$

where $\bar{\tau}=\int_{0}^{1} \tau d x=\int_{0}^{1} \tau_{0} d x$, constant $\bar{\theta}>0$ is determined by

$$
e(\bar{\tau}, \bar{\theta})=E_{0} \equiv \int_{0}^{1}\left(\frac{1}{2}\left(u_{0}^{2}+\left|\mathbf{w}_{0}\right|^{2}+\tau_{0}\left|\mathbf{b}_{0}\right|^{2}\right)+e\left(\tau_{0}, \theta_{0}\right)\right) d x .
$$

Proof See, e.g., Qin et al. [20, 21].

Lemma 2.3 Assume that the initial data $\left(\tau_{0}, u_{0}, \mathbf{w}_{0}, \mathbf{b}_{0}, \theta_{0}\right) \in H_{+}^{2}$ and compatibility conditions are satisfied, the heat conductivity $\kappa$ is a $C^{3}$ function satisfying $(2.1)$ on $0<\tau<\infty$ and $0 \leq \theta<\infty$, and there exists a constant $\varepsilon_{0}>0$ such that $\bar{\tau}=\int_{0}^{1} \tau_{0} d x \leq \varepsilon_{0}$. Then problem (1.1)-(1.8) admits a unique global solution $(\tau, u, \mathbf{w}, \mathbf{b}, \theta) \in H_{+}^{2}$ verifying that, for any $t>0$,

$$
\begin{aligned}
& \|\tau(t)-\bar{\tau}\|_{H^{2}}^{2}+\|u(t)\|_{H^{2}}^{2}+\|\mathbf{w}(t)\|_{H^{2}}^{2}+\|\mathbf{b}(t)\|_{H^{2}}^{2}+\|\theta(t)-\bar{\theta}\|_{H^{2}}^{2}+\left\|u_{t}(t)\right\|^{2}+\left\|\mathbf{w}_{t}(t)\right\|^{2} \\
& \quad+\left\|\mathbf{b}_{t}(t)\right\|^{2}+\left\|\theta_{t}(t)\right\|^{2}+\int_{0}^{t}\left(\|\tau-\bar{\tau}\|_{H^{2}}^{2}+\|u\|_{H^{3}}^{2}+\|\mathbf{w}\|_{H^{3}}^{2}+\|\mathbf{b}\|_{H^{3}}^{2}+\|\theta-\bar{\theta}\|_{H^{3}}^{2}\right. \\
& \left.\quad+\left\|u_{t x}\right\|^{2}+\left\|\mathbf{w}_{t x}\right\|^{2}+\left\|\mathbf{b}_{t x}\right\|^{2}+\left\|\theta_{t x}\right\|^{2}\right)(s) d s \leq C_{2} .
\end{aligned}
$$

Proof See, e.g., Qin et al. [20, 21].

Lemma 2.4 The unique generalized global solution $(\tau, u, \mathbf{w}, \mathbf{b}, \theta)$ in $H_{+}^{i}(i=1,2)$ to problem (1.1)-(1.8) defines a nonlinear $C_{0}$-semigroup $S(t)$ on $H_{+}^{i}$. 
Proof By Lemmas 2.2-2.3, we know that, for any $t>0$, the operator $S(t):\left(\tau_{0}, u_{0}, \mathbf{w}_{0}\right.$, $\left.\mathbf{b}_{0}, \theta_{0}\right) \in H_{+}^{i} \rightarrow(\tau, u, \mathbf{w}, \mathbf{b}, \theta) \in H_{+}^{i}(i=1,2)$ exists and, by the uniqueness of generalized global solutions, satisfies on $H_{+}^{1}$, for any $t_{1}, t_{2} \in[0, \infty)$,

$$
S\left(t_{1}+t_{2}\right)=S\left(t_{1}\right) S\left(t_{2}\right)=S\left(t_{2}\right) S\left(t_{2}\right) .
$$

Lemma 2.5 If $\left(\tau_{0}, u_{0}, \mathbf{w}_{0}, \mathbf{b}_{0}, \theta_{0}\right) \in H_{\delta}^{1}$, then the following estimates hold for any $t>0$ :

$$
\begin{aligned}
& \delta_{2} \leq \int_{0}^{1} \tau(x, t) d x=\int_{0}^{1} \tau_{0}(x) d x \leq \delta_{3}, \\
& \delta_{6} \leq \int_{0}^{1} E(x, t) d x=\int_{0}^{1} E(x, 0) d x \equiv E_{0} \leq \delta_{7}, \\
& \Phi(t)+\int_{0}^{t} V(s) d s \leq \delta_{1} .
\end{aligned}
$$

Proof We integrate (1.1) with respect to $x$ and $t$ and exploit the boundary conditions (1.8), we will end up with (2.5). Integrating $(1.5)$ over $Q_{t}:=(0,1) \times(0, t)$ and noting $(1.8)$, we get (2.6). The conservation law of total energy, (1.5), can be rewritten as

$$
e_{t}+p u_{x}=\left(\frac{\kappa \theta_{x}}{\tau}\right)_{x}+\frac{\lambda u_{x}^{2}+\mu\left|\mathbf{w}_{x}\right|^{2}+\nu\left|\mathbf{b}_{x}\right|^{2}}{\tau},
$$

i.e.,

$$
C_{v} \theta_{t}+4 a \tau \theta^{3} \theta_{t}+\frac{R \theta \tau_{t}}{\tau}+\frac{4 a}{3} \tau_{t} \theta^{4}=\left(\frac{\kappa \theta_{x}}{\tau}\right)_{x}+\frac{\lambda u_{x}^{2}+\mu\left|\mathbf{w}_{x}\right|^{2}+\nu\left|\mathbf{b}_{x}\right|^{2}}{\tau} .
$$

Multiplying (2.9) by $\theta^{-1}$, and integrating the resulting equation over $Q_{t}$, we get (2.7).

Lemma 2.6 If $\left(\tau_{0}, u_{0}, \mathbf{w}_{0}, \mathbf{b}_{0}, \theta_{0}\right) \in H_{\delta}^{1}$, then the following estimates hold for any $t>0$ :

$$
0<C_{\delta}^{-1} \leq \int_{0}^{1} \theta d x \leq C_{\delta}
$$

Proof It follows from (2.7) and the convexity of the function $-\log y$ that

$$
\int_{0}^{1} \theta d x-\log \int_{0}^{1} \theta d x-1 \leq \int_{0}^{1}(\theta-\log \theta-1) d x \leq C_{\delta}, \quad \forall t>0,
$$

which implies that there exist $b(t) \in[0,1]$ and two positive constants $r_{1}, r_{2}$ such that

$$
0<r_{1} \leq \int_{0}^{1} \theta d x=\theta(b(t), t) \leq r_{2}
$$

where $r_{i}=r_{i}(\delta)(i=1,2)$ are two positive roots of the equation $y-\log y-1=C_{\delta}$. Thus (2.10) follows from (2.11). The proof is complete.

Lemma 2.7 If $\left(\tau_{0}, u_{0}, \mathbf{w}_{0}, \mathbf{b}_{0}, \theta_{0}\right) \in H_{\delta}^{1}$, then the following estimates hold for any $t>0$ :

$$
0 \leq C_{\delta}^{-1} \leq \tau(x, t) \leq C_{\delta}, \quad \forall(x, t) \in[0,1] \times[0,+\infty)
$$


Proof See, e.g., Qin et al. [20, 21].

Lemma 2.8 If $\left(\tau_{0}, u_{0}, \mathbf{w}_{0}, \mathbf{b}_{0}, \theta_{0}\right) \in H_{\delta}^{1}$, then the following estimates hold for any $t>0$ :

$$
\begin{aligned}
& \theta_{*} \leq \bar{\theta} \leq \theta^{*}, \\
& 0 \leq C_{\delta}^{-1} \leq \theta(x, t), \quad \forall(x, t) \in[0,1] \times[0,+\infty),
\end{aligned}
$$

where $\theta_{*}=\min _{\tau \in\left[\delta_{2}, \delta_{3}\right], e \in\left[\delta_{6}, \delta_{7}\right]} \hat{\theta}(\tau, e), \theta^{*}=\max _{\tau \in\left[\delta_{2}, \delta_{3}\right], e \in\left[\delta_{6}, \delta_{7}\right]} \hat{\theta}(\tau, e)$.

Proof We first show that, for boundary conditions (1.8),

$$
\min _{\tau \in\left[\delta_{2}, \delta_{3}\right], e \in\left[\delta_{6}, \delta_{7}\right]} \leq \bar{\theta} \leq \max _{\tau \in\left[\delta_{2}, \delta_{3}\right], e \in\left[\delta_{6}, \delta_{7}\right]} \hat{\theta}(\tau, e) .
$$

In fact, it follows from (2.5)-(2.6) that

$$
\delta_{6} \leq \bar{e}:=e(\bar{\tau}, \bar{\theta}) \leq \delta_{7}, \quad \delta_{2} \leq \bar{\tau} \leq \delta_{3},
$$

which implies that $\bar{\theta}=\hat{\theta}(\bar{\tau}, \bar{\theta})$ and (2.15) holds.

We derive from Lemma 2.2 that there exists a large time $t_{0}>0$ such that

$$
\theta(x, t) \geq \frac{1}{2} \bar{\theta}>0, \quad \forall t \geq t_{0} .
$$

On the other hand, we put $\omega:=\frac{1}{\theta},(2.8)$ becomes

$$
e_{\theta} \omega_{t}=\left(\frac{\kappa \omega_{x}}{\tau}\right)_{x}+\frac{\tau p_{\theta}^{2}}{4 \lambda}-\left[\frac{2 \kappa \omega_{x}^{2}}{\tau \omega}+\frac{\omega^{2}}{\tau}\left(\mu\left|\mathbf{w}_{x}\right|^{2}+\nu\left|\mathbf{b}_{x}\right|^{2}\right)+\frac{\lambda \omega^{2}}{\tau}\left(u_{x}-\frac{\tau p_{\theta}}{2 \lambda \omega}\right)^{2}\right],
$$

which with (2.3) and (2.12) implies that there exists a positive constant $C_{1}$ such that

$$
\omega_{t} \leq \frac{1}{e_{\theta}}\left(\frac{\kappa \omega_{x}}{\tau}\right)_{x}+C_{1}
$$

Defining $\tilde{\omega}(x, t):=C_{1} t+\max _{[0,1]} \frac{1}{\theta_{0}(x)}-\omega(x, t)$ and a parabolic operator $\mathcal{L}:=-\frac{\partial}{\partial t}+$ $\frac{1}{e_{\theta}} \frac{\partial}{\partial x}\left(\frac{\kappa}{\tau} \frac{\partial}{\partial x}\right)$, we have a system

$$
\begin{aligned}
& \mathcal{L} \tilde{\omega} \leq 0, \quad \text { on } Q_{T}=[0,1] \times\left[0, t_{0}+1\right], \\
& \left.\tilde{\omega}\right|_{t=0} \geq 0, \quad \text { on }[0,1], \\
& \left.\tilde{\omega}_{x}\right|_{x=0,1}=0, \quad \text { on }\left[0, t_{0}+1\right] .
\end{aligned}
$$

The standard comparison argument implies

$$
\min _{(x, t) \in \overline{\mathrm{Q}}_{T}} \tilde{\omega}(x, t) \geq 0
$$

which gives, for any $(x, t) \in \bar{Q}_{T}$,

$$
\theta(x, t) \geq\left(C_{1} t+\max _{x \in[0,1]} \frac{1}{\theta_{0}(x)}\right)^{-1} .
$$


Thus,

$$
\theta(x, t) \geq\left(C_{1} t_{0}+\max _{x \in[0,1]} \frac{1}{\theta_{0}(x)}\right)^{-1} \geq C_{\theta}^{-1}, \quad 0 \leq t \leq t_{0}
$$

which, together with (2.12) and (2.16), gives (2.14).

Lemma 2.9 For initial data belonging to an arbitrary fixed bounded set $\mathcal{B}$ of $H_{\delta}^{1}$, there is $t_{0}>0$ depending only on the boundedness of this bounded set $\mathcal{B}$ such that, for all $t \geq t_{0}$, $x \in[0,1]$,

$$
\delta_{4} \leq \theta(x, t) \leq \delta_{5}, \quad \delta_{2} / 2 \leq \tau(x, t) \leq 2 \delta_{3} .
$$

Proof Suppose that the assertion in Lemma 2.9 is not true. Then there is a sequence $t_{n} \rightarrow$ $+\infty$, such that, for all $x \in[0,1]$,

$$
\sup \theta\left(x, t_{n}\right)>\delta_{5},
$$

where sup is taken for all initial data in a given bounded set $\mathcal{B}$ of $H_{\delta}^{1}$. Then there exists $\left(\tau_{0}, u_{0}, \mathbf{w}_{0}, \mathbf{b}_{0}, \theta_{0}\right) \in \mathcal{B}$ such that, for the corresponding solution $(\tau, u, \mathbf{w}, \mathbf{b}, \theta)$, we have

$$
\theta\left(x, t_{n}\right)>\delta_{5}, \quad x \in[0,1]
$$

which yields

$$
\bar{\theta} \geq \delta_{5} .
$$

This contradicts (2.13). Similarly, we can prove other parts of (2.17). The proof is complete.

Remark 2.10 It follows from Lemma 2.5 and Lemma 2.9 that, for initial data belonging to a given bounded set $\mathcal{B}$ of $H_{\delta}^{1}$, the orbit will re-enter $H_{\delta}^{1}$ and stay there after a finite time.

In the sequel, we shall prove the existence of an absorbing ball in $H_{\delta}^{1}$. Since we assume that the initial data $\left(\tau_{0}, u_{0}, \mathbf{w}_{0}, \mathbf{b}_{0}, \theta_{0}\right)$ belong to an arbitrary bounded set $\mathcal{B}$ of $H_{\delta}^{1}$, there is a positive constant $B$ such that $\left\|\left(\tau_{0}, u_{0}, \mathbf{w}_{0}, \mathbf{b}_{0}, \theta_{0}\right)\right\|_{H^{1}} \leq B$. We use $C_{B, \delta}$ to denote generic positive constants depending on $B$ and $\delta_{i}(i=1, \ldots, 7)$.

Lemma 2.11 For any initial data $\left(\tau_{0}, u_{0}, \mathbf{w}_{0}, \mathbf{b}_{0}, \theta_{0}\right) \in H_{\delta}^{1}$, the unique generalized global solution $(\tau, u, \mathbf{w}, \mathbf{b}, \theta)$ to problem (1.1)-(1.8) satisfies the estimate:

$$
\begin{aligned}
& \frac{1}{2}\left(u^{2}+|\mathbf{w}|^{2}+\tau|\mathbf{b}|^{2}\right)+C_{B, \delta}^{-1}\left(|\tau-\bar{\tau}|^{2}+|\eta-\bar{\eta}|^{2}\right) \\
& \quad \leq \mathcal{E}(\tau, u, \mathbf{w}, \mathbf{b}, \theta) \\
& \quad \leq \frac{1}{2}\left(u^{2}+|\mathbf{w}|^{2}+\tau|\mathbf{b}|^{2}\right)+C_{B, \delta}\left(|\tau-\bar{\tau}|^{2}+|\eta-\bar{\eta}|^{2}\right) .
\end{aligned}
$$

Proof See, e.g., Qin et al. [20, 21]. 
Lemma 2.12 There are positive constants $\gamma_{1}^{\prime}=\gamma_{1}^{\prime}\left(C_{B, \delta}\right)>0$ such that, for any fixed $\gamma \in$ $\left(0, \gamma_{1}^{\prime}\right]$ and for any $t>0$, we have

$$
\begin{gathered}
e^{\gamma t}\left(\|\tau(t)-\bar{\tau}\|^{2}+\|u(t)\|^{2}+\|\mathbf{w}(t)\|^{2}+\|\mathbf{b}(t)\|^{2}+\|\theta(t)-\bar{\theta}\|^{2}+\left\|\tau_{x}(t)\right\|^{2}+\left\|\rho_{x}(t)\right\|^{2}\right) \\
+\int_{0}^{t} e^{\gamma s}\left(\left\|\rho_{x}\right\|^{2}+\left\|u_{x}\right\|^{2}+\left\|\mathbf{w}_{x}\right\|^{2}+\left\|\mathbf{b}_{x}\right\|^{2}+\left\|\theta_{x}\right\|^{2}+\left\|\tau_{x}\right\|^{2}\right)(s) d s \leq C_{B, \delta} .
\end{gathered}
$$

Proof See, e.g., Qin et al. [20, 21].

Lemma 2.13 There exists a positive constant $\gamma_{1}=\gamma_{1}\left(C_{B, \delta}\right) \leq \gamma_{1}^{\prime}$ such that, for any $t>0$ and any fixed $\gamma \in\left(0, \gamma_{1}^{\prime}\right]$, the following estimate holds:

$$
\begin{gathered}
e^{\gamma t}\left(\left\|u_{x}(t)\right\|^{2}+\left\|\mathbf{w}_{x}(t)\right\|^{2}+\left\|\mathbf{b}_{x}(t)\right\|^{2}+\left\|\theta_{x}(t)\right\|^{2}\right)+\int_{0}^{t} e^{\gamma s}\left(\left\|u_{x x}\right\|^{2}+\left\|\mathbf{w}_{x x}\right\|^{2}\right. \\
\left.+\left\|\mathbf{b}_{x x}\right\|^{2}+\left\|\theta_{x x}\right\|^{2}+\left\|u_{t}\right\|^{2}+\left\|\mathbf{w}_{t}\right\|^{2}+\left\|\mathbf{b}_{t}\right\|^{2}+\left\|\theta_{t}\right\|^{2}\right)(s) d s \leq C_{B, \delta},
\end{gathered}
$$

which with Lemma 2.12 implies that, for any fixed $\gamma \in\left(0, \gamma_{1}^{\prime}\right]$,

$$
\|(\tau(t)-\bar{\tau}, u(t), \mathbf{w}(t), \mathbf{b}(t), \theta(t)-\bar{\theta})\| \leq C_{B, \delta} e^{-\gamma t} .
$$

Proof See, e.g., Qin et al. [20, 21].

Thus the following results on the existence of an absorbing set in $H_{\delta}^{1}$ follow from Lemma 2.13 .

Lemma 2.14 Let $R_{1}=R_{1}(\delta)=2 \sqrt{\delta_{3}^{2}+\left(\theta^{*}\right)^{2}}$ and

$$
B_{1}=\left\{(\tau(t), u(t), \mathbf{w}(t), \mathbf{b}(t), \theta(t)) \in H_{\delta}^{1},\|(\tau(t), u(t), \mathbf{w}(t), \mathbf{b}(t), \theta(t))\|_{H_{+}^{1}} \leq R_{1}\right\} .
$$

Then $B_{1}$ is an absorbing ball in $H_{\delta}^{1}$, i.e., there exists some

$$
t_{1}=t_{1}\left(C_{B, \delta}\right)=\max \left\{-\gamma_{1}^{-1} \log \left[2\left(\delta_{3}^{2}+\left(\theta^{*}\right)^{2}\right) / C_{B, \delta}\right], t_{0}\right\} \geq t_{0}
$$

such that when $t \geq t_{1},\|(\tau(t), u(t), \mathbf{w}(t), \mathbf{b}(t), \theta(t))\|_{H_{+}^{1}}^{2} \leq R_{1}^{2}$.

\section{An absorbing set in $\mathrm{H}^{2}$}

In this section we are going to prove the existence of an absorbing set in $H_{\delta}^{2}$. Throughout this section we always assume that the initial data belonging to an arbitrary fixed bounded set $\mathcal{B}$ of $H_{\delta}^{2}$.

The next two lemmas concern the existence of an absorbing set in $H_{\delta}^{2}$.

Lemma 3.1 There exists a positive constant $\gamma_{2}^{\prime}=\gamma_{2}^{\prime}\left(C_{B, \delta}\right) \leq \gamma_{1}$ such that, for any fixed $\gamma \in$ $\left(0, \gamma_{2}^{\prime}\right]$, the following estimate holds:

$$
\begin{aligned}
& e^{\gamma t}\left(\left\|u_{t}(t)\right\|^{2}+\left\|\mathbf{w}_{t}(t)\right\|^{2}+\left\|\mathbf{b}_{t}(t)\right\|^{2}+\left\|\theta_{t}(t)\right\|^{2}+\|u(t)\|_{H^{2}}^{2}+\|\mathbf{w}(t)\|_{H^{2}}^{2}\right. \\
& \left.\quad+\|\mathbf{b}(t)\|_{H^{2}}^{2}+\|\theta(t)\|_{H^{2}}^{2}\right)+\int_{0}^{t} e^{\gamma s}\left(\left\|u_{t x}\right\|^{2}+\left\|\mathbf{w}_{t x}\right\|^{2}+\left\|\mathbf{b}_{t x}\right\|^{2}+\left\|\theta_{t x}\right\|^{2}\right)(s) d s \\
& \leq C_{B, \delta}, \quad \forall t>0 .
\end{aligned}
$$


Proof See, e.g., Qin et al. [20, 21].

Lemma 3.2 There exists a positive constant $\gamma_{2}=\gamma_{2}\left(C_{B, \delta}\right) \leq \gamma_{2}^{\prime}$ such that, for any fixed $\gamma \in\left(0, \gamma_{2}\right]$, the following estimate holds:

$$
\|\tau(t)-\bar{\tau}\|_{H^{2}} \leq C_{B, \delta} e^{-\gamma t}
$$

which together with Lemma 3.1 implies that, for any $\gamma \in\left(0, \gamma_{2}\right]$ and $\forall t>0$,

$$
\begin{aligned}
& \|\tau(t)\|_{H^{2}}^{2}+\|u(t)\|_{H^{2}}^{2}+\|\mathbf{w}(t)\|_{H^{2}}^{2}+\|\mathbf{b}(t)\|_{H^{2}}^{2}+\|\theta(t)\|_{H^{2}}^{2} \\
& \quad \leq 2\left(\delta_{3}^{2}+\left(\theta^{*}\right)^{2}\right)+C_{B, \delta} e^{-\gamma t} .
\end{aligned}
$$

Proof See, e.g., Qin et al. [20, 21].

Now if we define $t_{2}=t_{2}\left(C_{B, \delta}\right) \geq \max \left(t_{1}\left(C_{B, \delta},-\gamma_{2}^{-1}\right) \log \left(2\left(\delta_{3}^{2}+\left(\theta^{*}\right)^{2}\right) / C_{B, \delta}\right)\right)$, then estimate (3.3) implies that, for any $t \geq t_{2}\left(C_{B, \delta}\right)$,

$$
\left.\|\tau(t)\|_{H^{2}}^{2}+\|u(t)\|_{H^{2}}^{2}+\|\mathbf{w}(t)\|_{H^{2}}^{2}+\|\mathbf{b}(t)\|_{H^{2}}^{2}+\|\theta(t)\|_{H^{2}}^{2} \leq 4\left(\delta_{3}^{2}+\left(\theta^{*}\right)^{2}\right)\right) .
$$

Taking $R_{2}=2 \sqrt{\delta_{3}^{2}+\left(\theta^{*}\right)^{2}}$, we immediately infer the following theorem.

Theorem 3.3 The ball $B_{2}=\left\{(\tau(t), u(t), \mathbf{w}(t), \mathbf{b}(t), \theta(t)) \in H_{\delta}^{2}, \|(\tau(t), u(t), \mathbf{w}(t), \mathbf{b}(t)\right.$, $\left.\theta(t)) \|_{H_{+}^{2}}^{2} \leq R_{2}^{2}\right\}$ is an absorbing ball in $H_{\delta}^{2}$, i.e., when $t \geq t_{2}$, we have

$$
\|(\tau(t), u(t), \mathbf{w}(t), \mathbf{b}(t), \theta(t))\|_{H_{+}^{2}}^{2} \leq R_{2}^{2}
$$

\section{Universal attractor in $H^{1}$ and $H^{2}$}

In this section we finish the proof of Theorems 1.1 and 1.2. Having proved the existence of absorbing balls in $H_{\delta}^{2}$ and $H_{\delta}^{1}$, we can use the abstract framework established in [25] by Ghidaglia (see also Lemma 2.1) to conclude that

Lemma 4.1 The set

$$
\omega\left(B_{2}\right)=\bigcap_{s \geq 0} \overline{\bigcup_{t \geq s} S(t) B_{2}},
$$

where the closures are taken with respect to the weak topology of $H_{+}^{2}$, is included in $B_{2}$ and is nonempty. It is invariant by $S(t)$, i.e.,

$$
S(t) \omega\left(B_{2}\right)=\omega\left(B_{2}\right), \quad \forall t>0 .
$$

Lemma 4.2 The set

$$
\mathcal{A}_{2, \delta}=\omega\left(B_{2}\right)
$$

satisfies

$$
\mathcal{A}_{2, \delta} \text { is bounded and weakly closed in } H_{\delta}^{2} \text {, }
$$




$$
S(t) \mathcal{A}_{2, \delta}=\mathcal{A}_{2, \delta}, \quad \forall t \geq 0,
$$

for every bounded set $\mathcal{B}$ in $H_{\delta}^{2}$,

$$
\lim _{t \rightarrow+\infty} d^{\omega}\left(S(t) \mathcal{B}, \mathcal{A}_{2, \delta}\right)=0
$$

Moreover, it is the maximal set in the sense of an inclusion that satisfies (4.4), (4.5) and (4.6).

Proof The proofs of Lemmas 4.1 and 4.2 follow from Lemma 2.1, using the facts that $S(t)$ is continuous on $H_{\delta}^{2}$ and $H_{\delta}^{1}$, respectively, $H_{\delta}^{2}$ is compactly embedded in $H_{\delta}^{1}, B_{2}$, and $B_{1}$ are absorbing balls in $H_{\delta}^{2}$ and $H_{\delta}^{1}$, respectively.

Following [25], we also call $\mathcal{A}_{2, \delta}$ the universal attractor of $S(t)$ in $H_{\delta}^{2}$. In order to discuss the existence of a universal attractor in $H_{\delta}^{1}$, we need to prove the following lemma.

Lemma 4.3 For every $t \geq 0$, the mapping $S(t)$ is continuous on bounded sets of $H_{\delta}^{1}$ for the topology induced by the norm $\left(L^{2}\right)^{7}$.

Proof We suppose that $\left(\tau_{0 j}, u_{0 j}, \mathbf{w}_{0 j}, \mathbf{b}_{0 j}, \theta_{0 j}\right) \in H_{\delta}^{1},\left\|\left(\tau_{0 j}, u_{0 j}, \mathbf{w}_{0 j}, \mathbf{b}_{0 j}, \theta_{0 j}\right)\right\|_{H^{1}} \leq R,\left(\tau_{j}, u_{j}, \mathbf{w}_{j}\right.$, $\left.\mathbf{b}_{j}, \theta_{j}\right)=S(t)\left(\tau_{0 j}, u_{0 j}, \mathbf{w}_{0 j}, \mathbf{b}_{0 j}, \theta_{0 j}\right)$, and $(\tau, u, \mathbf{w}, \mathbf{b}, \theta)=\left(\tau_{1}, u_{1}, \mathbf{w}_{1}, \mathbf{b}_{1}, \theta_{1}\right)-\left(\tau_{2}, u_{2}, \mathbf{w}_{2}, \mathbf{b}_{2}, \theta_{2}\right)$.

Subtracting the corresponding equations (1.1)-(1.5) satisfied by $\left(\tau_{1}, u_{1}, \mathbf{w}_{1}, \mathbf{b}_{1}, \theta_{1}\right)$ and $\left(\tau_{2}, u_{2}, \mathbf{w}_{2}, \mathbf{b}_{2}, \theta_{2}\right)$, we obtain

$$
\begin{aligned}
\tau_{t}= & u_{x} \\
u_{t}= & -\frac{1}{2}|\mathbf{b}|_{x}^{2}-\left(\mathbf{b}_{1} \mathbf{b}_{2 x}+\mathbf{b}_{2} \mathbf{b}_{1 x}\right)+R\left[\frac{\theta \tau_{1 x}}{\tau_{1}^{2}}+\left(\frac{\theta_{2} \tau}{\tau_{1} \tau_{2}}\right)_{x}-\frac{\theta_{x}}{\tau_{1}}\right] \\
& -\lambda\left[\frac{u_{x} \tau_{1 x}}{\tau_{1}^{2}}+\left(\frac{u_{2 x} \tau}{\tau_{1} \tau_{2}}\right)_{x}-\frac{u_{x x}}{\tau_{1}}\right]-\frac{4 a}{3}\left(\theta_{1}^{3} \theta_{1 x}-\theta_{2}^{3} \theta_{2 x}\right) \theta_{x}^{4}, \\
\mathbf{w}_{t}= & -\mathbf{b}_{x}-\mu\left[\frac{\mathbf{w}_{x} \tau_{1 x}}{\tau_{1}^{2}}+\left(\frac{\mathbf{w}_{2 x} \tau}{\tau_{1} \tau_{2}}\right)_{x}-\frac{\mathbf{w}_{x x}}{\tau_{1}}\right], \\
\tau \mathbf{b}_{t}= & -u_{x} \mathbf{b}-\mathbf{w}_{x}-v\left[\frac{\mathbf{b}_{x} \tau_{1 x}}{\tau_{1}^{2}}+\left(\frac{\mathbf{b}_{2 x} \tau}{\tau_{1} \tau_{2}}\right)-\frac{\mathbf{b}_{x x}}{\tau_{1}}\right], \\
C_{v} \theta_{t}= & -\kappa\left[\frac{\theta_{x} \tau_{1 x}}{\tau_{1}^{2}}+\left(\frac{\theta_{2 x} \tau}{\tau_{1} \tau_{2}}\right)_{x}-\frac{\theta_{x x}}{\tau_{1}}\right]+\frac{\lambda u_{1 x}^{2}+\mu\left|\mathbf{w}_{1 x}\right|^{2}+v\left|\mathbf{b}_{1 x}\right|^{2}}{\tau_{1}} \\
& -\frac{\lambda u_{2 x}^{2}+\mu\left|\mathbf{w}_{2 x}\right|^{2}+v\left|\mathbf{b}_{2 x}\right|^{2}}{\tau_{2}}-4 a \tau_{1} \theta_{1}^{3} \theta_{1 t}+\frac{R \theta_{1} \tau_{1 t}}{\tau_{1}}+\frac{4 a}{3} \tau_{1 t} \theta_{1}^{4} \\
& +4 a \tau_{2} \theta_{2}^{3} \theta_{2 t}+\frac{R \theta_{2} \tau_{2 t}}{\tau_{2}}+\frac{4 a}{3} \tau_{2 t} \theta_{2}^{4} .
\end{aligned}
$$

By Lemma 2.3, we know that, for any $t>0$ and $j=1,2$,

$$
\begin{aligned}
& \left\|\tau_{j}(t), u_{j}(t), \mathbf{w}_{j}(t), \mathbf{b}_{j}(t), \theta_{j}(t)\right\|_{H^{1}}^{2}+\int_{0}^{t}\left(\left\|\tau_{j x}\right\|^{2}+\left\|u_{j x}\right\|_{H^{2}}^{2}+\left\|\mathbf{w}_{j x}\right\|_{H^{1}}^{2}+\left\|\mathbf{b}_{j x}\right\|_{H^{1}}^{2}\right. \\
& \left.+\left\|\theta_{j x}\right\|_{H^{1}}^{2}+\left\|u_{j t}\right\|^{2}+\left\|\mathbf{w}_{j t}\right\|^{2}+\left\|\mathbf{b}_{j t}\right\|^{2}+\left\|\theta_{j t}\right\|^{2}\right)(s) d s \leq C_{R, \delta},
\end{aligned}
$$

where $C_{R, \delta}>0$ is a constant depending only on $R$ and $\delta$. 
Multiplying (1.1)-(1.5) by $\tau, u, \mathbf{w}, \mathbf{b}$, and $\theta$, respectively, adding them up and integrating the result over $[0,1]$, and using Lemmas 2.2-2.3, the Cauchy inequality, the embedding theorem, the mean value theorem, and the inequalities

$$
\|\theta\|_{L^{\infty}}^{2} \leq C\left(\|\theta\|\left\|\theta_{x}\right\|+\|\theta\|^{2}\right), \quad\|\tau\|_{L^{\infty}}^{2} \leq C\left\|\tau_{x}\right\|
$$

we deduce that, for any small $\epsilon>0$,

$$
\begin{aligned}
& \frac{d}{d t}\left(\|\tau\|^{2}+\|u\|^{2}+\|\mathbf{w}\|^{2}+\|\mathbf{b}\|^{2}+\|\theta\|^{2}\right)+\int_{0}^{1}\left(\frac{\kappa \theta_{x}^{2}}{\tau \theta^{2}}+\frac{\lambda u_{x}^{2}+\mu\left|\mathbf{w}_{x}\right|^{2}+v\left|\mathbf{b}_{x}\right|^{2}}{\tau \theta}\right)(x, t) d x \\
& \leq \epsilon\left(\left\|u_{x}\right\|^{2}+\left\|\mathbf{w}_{x}\right\|^{2}+\left\|\mathbf{b}_{x}\right\|^{2}+\left\|\theta_{x}\right\|^{2}\right) \\
& \quad+C_{R, \delta}(\epsilon) H(t)\left(\|\tau\|^{2}+\|u\|^{2}+\|\mathbf{w}\|^{2}+\|\mathbf{b}\|^{2}+\|\theta\|^{2}\right)
\end{aligned}
$$

which, together with Lemmas 2.5-2.9, yields

$$
\begin{aligned}
& \frac{d}{d t}\left(\|\tau\|^{2}+\|u\|^{2}+\|\mathbf{w}\|^{2}+\|\mathbf{b}\|^{2}+\|\theta\|^{2}\right)+C_{\delta}^{-1}\left(\left\|u_{x}\right\|^{2}+\left\|\mathbf{w}_{x}\right\|^{2}+\left\|\mathbf{b}_{x}\right\|^{2}+\left\|\theta_{x}\right\|^{2}\right) \\
& \quad \leq C_{R, \delta} H(t)\left(\|\tau\|^{2}+\|u\|^{2}+\|\mathbf{w}\|^{2}+\|\mathbf{b}\|^{2}+\|\theta\|^{2}\right)
\end{aligned}
$$

where by (4.12), $H(t)=\left\|\theta_{1 t}\right\|^{2}+\left\|\theta_{2 t}\right\|^{2}+\left\|u_{1 x x}\right\|^{2}+\left\|u_{2 x x}\right\|^{2}\|+\| \mathbf{w}_{1 x x}\left\|^{2}+\right\| \mathbf{w}_{2 x x}\left\|^{2}\right\|+\left\|\mathbf{b}_{1 x x}\right\|^{2}+$ $\left\|\mathbf{b}_{2 x x}\right\|^{2}\|+\| \theta_{1 x x}\left\|^{2}+\right\| \theta_{2 x x} \|^{2}+1$ satisfies, for any $t>0$,

$$
\int_{0}^{t} H(s) d s \leq C_{R, \delta}(1+t) .
$$

Therefore the assertion of this lemma follows from Gronwall's inequality, (4.13), and (4.14). The proof is complete.

Now we can again use Lemma 2.1 to obtain the following result on existence of a universal attractor in $H_{\delta}^{1}$.

Lemma 4.4 The set

$$
\mathcal{A}_{1, \delta}=\bigcap_{s \geq 0} \overline{\bigcup_{t \geq s} S(t) B_{1}},
$$

where the closures are taken with respect to the weak topology of $H_{+}^{1}$ is the (maximal) universal attractor in $H_{\delta}^{1}$.

Remark 4.5 Since $\mathcal{A}_{2, \delta}$ is bounded in $H_{+}^{2}$ and is bounded in $H_{+}^{1}$ and by the invariance property (4.5), we have

$$
\mathcal{A}_{2, \delta} \subset \mathcal{A}_{1, \delta}
$$

On the contrary if we knew that $\mathcal{A}_{1, \delta}$ is bounded in $H_{+}^{2}$, then the opposite inclusion would hold.

Proof of Theorems 1.1 and 1.2 Combing Lemmas 2.1-2.4 and Lemmas 4.1-4.4, we easily complete the proofs of Theorems 1.1 and 1.2. 


\section{Competing interests}

The author declares to have no competing interests.

\section{Acknowledgements}

The work was in part supported by the NNSF of China (No. 11326158 and No. 11271066).

Received: 1 December 2014 Accepted: 10 August 2015 Published online: 02 September 2015

\section{References}

1. Ducomet, B: A model of thermal dissipation for a one-dimensional viscous reactive and radiative. Math. Methods Appl. Sci. 22, 1323-1349 (1999)

2. Kazhikhov, AV: Sur la solubilité globale des problémes monodimensionnels aux valeurs initiales-limités pour les équations d'un gaz visqueux et calorifére. C. R. Acad. Sci. Paris Ser. A 284, 317-320 (1977)

3. Kazhikhov, AV: To a theory of boundary value problems for equations of one-dimensional nonstationary motion of viscous heat-conduction gases. In: Boundary Value Problems for Hydrodynamical Equations. Inst. Hydrodynamics, Siberian Branch Akad., USSR., vol. 50, pp. 37-62 (1981) (in Russian)

4. Kazhikhov, AV, Sheluhin, V: Unique global solution with respect to time of the initial-boundary value problems for one-dimensional equations of a viscous gas. J. Appl. Math. Mech. 41, 273-282 (1977)

5. Kawashima, S, Nishida, T: Global solutions to the initial value problem for the equations of one-dimensional motion of viscous polytropic gases. J. Math. Kyoto Univ. 21, 825-837 (1981)

6. Zheng, S, Qin, Y: Universal attractor for the Navier-Stocks equations of compressible and heat-conductive fluid in bounded annular domains in $R^{n}$. Arch. Ration. Mech. Anal. 160, 153-179 (2001)

7. Kawohl, B: Global existence of large solutions to initial boundary value problems for the equations of one-dimensional motion of viscous polytropic gases. J. Differ. Equ. 58, 76-103 (1985)

8. Jiang, S: On initial boundary value problems for a viscous heat-conducting one-dimensional real gas. J. Differ. Equ. 110, 157-181 (1994)

9. Qin, Y: Nonlinear Parabolic-Hyperbolic Coupled Systems and Their Attractors. Operator Theory, Advances in PDEs, vol. 184. Birkhäuser, Basel (2008)

10. Umehara, M, Tani, A: Global solution to the one-dimensional equations for a self-gravitating viscous radiative and reactive gas. J. Differ. Equ. 234, 439-463 (2007)

11. Qin, Y, Hu, G, Wang, T: Global smooth solutions for the compressible viscous and heat-conductive gas. Q. Appl. Math. 69, 509-528 (2011)

12. Chen, G-Q, Wang, D: Global solutions of nonlinear magnetohydrodynamics with large initial data. J. Differ. Equ. 182 344-376 (2002)

13. Chen, G-Q, Wang, D: Existence and continuous dependence of large solutions for the magnetohydrodynamics equations. Z. Angew. Math. Phys. 54, 608-632 (2003)

14. Hoff, D, Tsyganov, E: Uniqueness and continuous dependence of weak solutions in compressible magnetohydrodynamics. Z. Angew. Math. Phys. 56, 791-840 (2005)

15. Fan, J, Jiang, S, Nakamura, G: Stability of weak solutions to equations of magnetohydrodynamics with Lebesgue initia data. J. Differ. Equ. 251, 2025-2036 (2011)

16. Fan, J, Jiang, S, Nakamura, G: Vanishing sheer viscosity limit in the magnetohydrodynamic equations. Commun. Math Phys. 270, 691-708 (2007)

17. Vol'pert, Al, Hudjaev, SI: On the Cauchy problem for composite systems of nonlinear differential equations. Math. USSR Sb. 16, 517-544 (1972)

18. Wang, D: Large solutions to the initial-boundary value problem for planar magnetohydrodynamics. SIAM J. Appl. Math. 63, 1424-1441 (2003)

19. Ströhmer, G: About compressible viscous fluid flow in a bounded region. Pac. J. Math. 143, 359-375 (1990)

20. Qin, Y, Liu, X, Yang, X: Global existence and exponential stability for a $1 D$ compressible and radiative MHD flow. J. Differ. Equ. 253, 1439-1488 (2012)

21. Qin, Y, Liu, X, Wang, T: Global Existence and Uniqueness of Nonlinear Evolutionary Fluid Equations. Frontiers in Mathematics. Birkhäuser, Basel (2015)

22. Ducomet, B, Feireisl, E: The equations of magnetohydrodynamics: on the interaction between matter and radiation in the evolution of gaseous stars. Commun. Math. Phys. 266, 595-629 (2006)

23. Zhang, J, Xie, F: Global solution for a one-dimensional model problem in thermally radiative magnetohydrodynamics. J. Differ. Equ. 245, 1853-1882 (2008)

24. Teman, R: Infinite-Dimensional Dynamical Systems in Mechanics and Physics. Applied Mathematical Science, vol. 68. Springer, New York (1988)

25. Ghidaglia, JM: Finite dimensional behavior for weakly damped driven Schrödinger equations. Ann. Inst. Henri Poincaré 5, 365-405 (1988) 